Antonio Carroccio, Ignazio Brusca, Pasquale Mansueto*, Alberto D’alcamo, Maria Barrale, Maurizio Soresi, Aurelio Seidita, Stella M. La Chiusa, Giuseppe lacono and Delia Sprini

\title{
A comparison between two different in vitro basophil activation tests for gluten- and cow's milk protein sensitivity in irritable bowel syndrome (IBS)-like patients
}

\section{Abstract}

Background: The diagnosis of food hypersensitivity (FH) in adult patients with gastrointestinal symptoms, beyond the immediate IgE-mediated clinical manifestations, is very often difficult. The aims of our study were to: 1) evaluate the frequency of $\mathrm{FH}$ in patients with irritable bowel syndrome (IBS)-like clinical presentation; and 2) compare the diagnostic accuracy of two different methods of in vitro basophil activation tests.

Methods: Three hundred and five patients, (235 females, age range 18-66 years) were included and underwent a diagnostic elimination diet and successive double-blind placebo-controlled (DBPC) challenges. Two different methods of in vitro basophil activation tests (BAT) (CD63 expression after in vitro wheat or cow's milk protein stimulation) were evaluated: one was performed on separated leukocytes, and the other on whole blood.

Results: Ninety patients of the 305 studied (29.5\%) were positive to the challenges and were diagnosed as suffering from FH. BAT on separate leukocytes showed a sensitivity of $86 \%$ and a specificity of $91 \%$ in $\mathrm{FH}$ diagnosis. BAT on whole blood showed a sensitivity of 15\%-20\% and a specificity of $73 \%$ in $\mathrm{FH}$ diagnosis $(\mathrm{p}<0.0001$ compared to the other method).

Conclusions: About one third of the IBS patients included in the study were suffering from $\mathrm{FH}$ and were cured on the elimination diet. The BAT based on CD63 detection on whole blood samples did not work in FH diagnosis and showed a significantly lower sensitivity, specificity and diagnostic accuracy than the assay based on separated leukocytes.

Keywords: basophils activation test; cytofluorimetry; food hypersensitivity; gluten sensitivity; irritable bowel syndrome.
*Corresponding author: Dott. Pasquale Mansueto, MD, Department of Internal Medicine and Specialist (DIMIS), Via del Vespro 129, 90129 Palermo, Italy, Phone: +39 0916554337 ,

E-mail: pasquale.mansueto@unipa.it

Antonio Carroccio: Internal Medicine, Giovanni Paolo II Hospital, Sciacca and University of Palermo, Palermo, Italy Ignazio Brusca, Maria Barrale and Stella M. La Chiusa: Laboratory Medicine, Buccheri La Ferla Hospital, Palermo, Italy Pasquale Mansueto, Alberto D’alcamo, Maurizio Soresi, Aurelio Seidita, Giuseppe Iacono and Delia Sprini: Internal Medicine, University Hospital of Palermo, Palermo, Italy

\section{Introduction}

The diagnosis of food hypersensitivity (FH) in adult patients with gastrointestinal symptoms, beyond the immediate IgE-mediated clinical manifestations, is very often difficult. The recent US guidelines recommended that the diagnosis of IgE-mediated $\mathrm{FH}$ requires both the presence of allergen-specific IgE and the development of specific symptoms on exposure to that food [1]. However, it is well known that most of the gastrointestinal symptoms due to suspect FH lack an IgE-mediated mechanism $[2,3]$ and in these cases no laboratory assay is considered useful to make the diagnosis $[1,3]$.

Patients presenting with irritable bowel syndrome (IBS) who are suspected of FH are included in this difficult diagnostic category. There is general agreement that some of these patients are really affected with $\mathrm{FH}$ [2] as demonstrated by several studies [4-6], but no immunology assay is helpful in the diagnosis. A double-blind placebo controlled (DBPC) challenge is the gold standard recommended to confirm the suspicion.

Very recently, we showed that a cytological assay based on in vitro basophil activation after food-antigen stimulation had a sensitivity of $86 \%$ in the diagnosis of FH in patients with IBS-like symptoms [7]. 
confirm the

running

title

The present study included patients with IBS diagnosis and suspected FH. The aims of the study were to: 1) evaluate the frequency of FH in patients with IBS-like clinical presentation; and 2) compare the diagnostic accuracy of two different methods of in vitro basophil activation tests (BAT).

\section{Materials and methods}

\section{Study design}

The study design contained two parts. In the first retrospective part, we included all patients with IBS diagnosis who had undergone DBPC challenges for suspected $\mathrm{FH}$, consecutively referred as outpatients to the Department of Internal Medicine of the University of Palermo or the Internal Medicine of the Hospital of Sciacca, Italy from January 2007 to June 2011. The inclusion criteria were: 1) age $>17$ years; 2) diagnosis of IBS; 3) suspected FH diagnosis evaluated by means of DBPC challenges; 4) follow-up duration longer than 12 months after the initial diagnosis; and 5) >1 outpatient visits during the follow-up period.

Exclusion criteria were: 1) patients with a diagnosis of organic gastrointestinal disease; 2) lack of follow-up visits after the initial diagnosis; and 3) lack of DBPC-challenge method in the FH diagnosis.

IBS diagnosis was based on the Rome II criteria for functional gastrointestinal disorders [8]. Furthermore, organic gastrointestinal disorders were excluded by an accurate work-up, as previously described [7].

In the second prospective part of the study we called back 20 patients ( 15 female, 5 male): 10 of these patients had tested positive at the DBPC challenges and 10 had tested negative; they were chosen at random from those previously included in the first part of the study. All these patients during the first part of the study had undergone the in vitro BAT based on the demonstration of altered membrane phenotypes on allergen-activated basophil, with cytofluorometric detection of CD63 protein, after leukocyte separation. During the second part of the study they underwent the in vitro BAT based on the same assay performed on whole blood; furthermore, they underwent open food challenges again.

None of the enrolled patients were on any medication or were undergoing the elimination diet at the time of the first part of the study. They were asked to suspend medications and/or diet at least 3 weeks before the beginning of the study protocol.

The study was conducted in accordance with the principles of the Declaration of Helsinki. The study protocol was approved by the Ethics Committee of the University Hospital of Palermo and all patients gave their informed consent to participate.

\section{Flow cytometric basophil activation test}

After enrolment in the first part of the study, the patients underwent flow cytometric BAT. The assay was performed by different operators, unaware of the clinical history of the patients and the results of the other tests.
From January 2007 to March 2010, the BAT was based for the detection of the surface CD63 protein, after centrifugation and leukocyte separation (230 patients: 170 females, median age 33.5 years). From April 2010 to June 2011 the detection of the surface CD63 protein was performed on whole blood samples ( 75 patients: 65 females, median age 34 years). Both assays were performed with Flow-CAST ${ }^{\circledR}$ (Bühlmann Laboratories AG, Schönenbuch, Switzerland), according to the manufacturer's instructions.

\section{Flow cytometric basophil activation test - CD63 detection on separate leukocytes (old test)}

The manufacturer's allergens used in all patients were $\alpha$-lactalbumin, $\beta$-lactoglobulin, casein, and wheat. As a positive control, we used a monoclonal Le27 anti-IgE antibody solution (Bühlmann Laboratories AG). As a negative control, only the stimulation buffer containing IL-3 was used.

The blood tubes were centrifuged at $200 \mathrm{~g}$ for $5 \mathrm{~min}$ at $4^{\circ} \mathrm{C}$. The supernatant containing leukocytes was transferred by pipette into polypropylene tubes and centrifuged again at $500 \mathrm{~g}$ for $10 \mathrm{~min}$ at $4^{\circ} \mathrm{C}$. It was then decanted and the cell pellet was re-suspended in $100 \mu \mathrm{L}$ of stimulation buffer containing interleukin (IL) 3. The $50 \mu \mathrm{L}$ of reconstituted solutions of allergens were added to $50 \mu \mathrm{L}$ of cell suspension and the tubes incubated for $40 \mathrm{~min}$ at $37^{\circ} \mathrm{C}$. The reaction was stopped by adding $100 \mu \mathrm{L}$ of cold HEPES buffer ( $\mathrm{pH}$ 7.3) containing EDTA (HEPES 20 mM, NaCl 133 mM, KCl 5 mM, EDTA 0.27 mM) but with no calcium or magnesium (washing buffer) and the tubes were centrifuged at $1000 \mathrm{~g}$ for $5 \mathrm{~min}$ at $4^{\circ} \mathrm{C}$. The basophils from the cell pellet were double-labeled by adding $20 \mu \mathrm{L}$ of a mixture of anti-CD63 phycoerythrin-labeled antibody and anti-IgE fluorescein isothiocyanate (FITC)-labeled antibody. After the incubation for $30 \mathrm{~min}$ at $4^{\circ} \mathrm{C}$ (protected from light exposure), $4 \mathrm{~mL}$ of a pre-warmed $\left(18-28^{\circ} \mathrm{C}\right.$ ) erythrolytic reagent (lysing reagent) was added to each tube and left at room temperature for $5 \pm 1 \mathrm{~min}$. Cell lysis was stopped with $1 \mathrm{~mL}$ of washing buffer, the tubes centrifuged for $5 \mathrm{~min}$ at $1000 \mathrm{~g}$, the supernatants were decanted and $500 \mu \mathrm{L}$ of washing buffer added to each tube. The tubes were gently shaken before flow-cytometric analysis that was performed at once. In each assay, at least 500 basophils were counted.

In order to define a result as positive, the percentage of basophils activated after incubation with antigen should be at least threefold the percentage of basophils activated in the background tube. When the percentage of basophils activated spontaneously was below $2.5 \%$, we required an additional condition, namely that the percentage of basophils activated after contact with the antigen should be $\geq 5 \%$. These cut-offs were chosen on the basis of receiver operating characteristic (ROC) curves plotted in our laboratory, enabling us to achieve the highest possible sensitivity with optimal specificity.

The intra-assay variation of the test was $3 \%$ and was calculated from four blood samples taken from two healthy controls and two patients suffering from food allergy stimulated with stimulation buffer and anti-FceRI Ab and consecutively analyzed 10 times by flow cytometry. The inter-assay variation of the test was $6.5 \%$ and was calculated from the blood samples of three healthy controls and three patients suffering from food allergy stimulated with stimulation buffer and anti-FceRI Ab, analyzed 10 times by flow cytometry and re-tested after 2 weeks. 
The reproducibility of the assay had been previously reported to be excellent: the correlation coefficient, evaluated in 20 patients by three expert operators, was equal to $0.92(\mathrm{p}<0.001)$ [7].

\section{Flow cytometric basophil activation test - CD63 detection on whole blood (new test)}

The blood collected into tubes with EDTA was not centrifuged. Fifty microliters of patient's whole blood were added to $3.5 \mathrm{~mL}$ polypropylene tubes for each patient and for each allergen tested and controls, then $50 \mu \mathrm{L}$ of the corresponding stimulus were transferred by pipette, into $100 \mu \mathrm{L}$ of stimulation buffer and $20 \mu \mathrm{L}$ staining reagent (antiCD63 phycoerythrin with anti-CCR3 fluorescein isothiocyanate). The tubes were incubated for $15 \mathrm{~min}$ at $37^{\circ} \mathrm{C}$ in a water bath. After the incubation $3.5 \mathrm{~mL}$ of a pre-warmed $\left(18-28^{\circ} \mathrm{C}\right)$ erythrolytic reagent (lysing reagent) was added to each tube and left at room temperature for $5-10 \mathrm{~min}$. The tubes were centrifuged for $5 \mathrm{~min}$ at $500 \mathrm{~g}$, the supernatants were decanted, and $500 \mu \mathrm{L}$ of washing buffer added to each tube. The tubes were gently shaken before flow-cytometric analysis that was performed at once. In each assay, at least 500 basophils were counted. According to the old method of defining a result as positive, the percentage of basophils activated after incubation with antigen should be at least three-fold the percentage of basophils activated in the background tube. When the percentage of basophils activated spontaneously was $<2.5 \%$, we required an additional condition, namely that the percentage of basophils activated after contact with the antigen should be $\geq 5 \%$.

\section{Wheat and cow's milk proteins hypersensitivity diagnoses}

At entry to the study, those patients who had self-reduced wheat and/ or cow's milk consumption were invited to assume a minimum quantity of $30 \mathrm{~g}$ of wheat and $150 \mathrm{~mL}$ of cow's milk daily and were observed for 2-4 weeks on a regular diet. All patients then commenced a standard elimination diet, based on our previous experience [7, 9], with the exclusion of wheat, cow's milk, eggs, tomato and chocolate. Patients self-reporting FH were also asked to avoid ingestion and/or contact with the food(s) causing symptoms.

Furthermore, food diaries were maintained by the patients during the above periods to assess dietary intake and adherence to the diet.

After 4 weeks on the elimination diet, those patients who became asymptomatic underwent DBPC challenges. The challenges were performed with the reintroduction of a single food at a time; details about DBPC challenges have been previously published $[7,10]$. In the case of wheat, the DBPC challenge was performed with capsules coded A or B containing wheat or xylose, respectively. Capsules A or B were given for 2 consecutive weeks and then after 1 week of washout the patients received the other capsules for another 2 weeks (cross-over design).

During all the phases of the study, including the challenge period, the severity of symptoms was recorded: the patients completed a $100-\mathrm{mm}$ visual analog scale, with 0 representing no symptoms, which assessed overall symptoms and the specific symptoms they each reported.
DBPC challenge for cow's milk proteins was performed with an identical method, according to a standard protocol [7, 10], at least 4 weeks before or after wheat challenge and when the VAS score was $<10$, on the elimination diet.

The challenges were stopped when clinical reactions occurred (increase in VAS score >30) for at least 2 consecutive days (onset of abdominal discomfort or pain, associated with a change in stool frequency and/or appearance). The challenges were considered positive if the same symptoms which had been initially presented reappeared after their disappearance on the elimination diet.

After FH had been excluded or confirmed, all IBS patients were invited to continue the follow-up with regular visits every 6 months for 1 year.

\section{Statistical analysis}

We followed the STARD checklist for studies on the diagnostic accuracy of tests [11]. Tables were constructed for frequency and percentage. The sensitivity, the specificity and the diagnostic accuracy of the immunologic assays, along with their 95\% confidence intervals, were calculated by standard statistical methods [12]. The Fisher's test was used to compare the frequencies. To minimize type I errors, $\mathrm{p}<0.05$ was considered statistically significant. All statistical analyses were performed using the SPSS 11.0 statistical package (Systat Software Inc., CA, USA).

\section{Results}

\section{First part of the study}

Three hundred and five patients, (235 females, 70 males, age range 18-66 years, median age 34 years), fulfilled the inclusion criteria and were studied. Of these, 105 became asymptomatic on the elimination diet (VAS score $<10$ ) and underwent DBPC challenges.

Figure 1 Summary of the challenges results. Seventy patients were positive to both wheat and cow's milk protein challenge (increase in VAS score $>30$ ), 12 patients were positive to wheat challenge only and eight patients were positive to cow's milk protein challenge only. In total, 90 patients of the 305 studied (29.5\%) were positive to the challenges and were diagnosed as suffering from $\mathrm{FH}$ (FH group). In contrast, a diagnosis of IBS unrelated to $\mathrm{FH}$ was made in the remaining 215 patients (IBS group).

Table 2 shows the results of the fluorometric CD63 assays on separate leukocytes and on whole blood in the IBS and in the FH patients. According to these results, Table 3 shows the sensitivity, specificity and diagnostic accuracy of the two assays in the diagnosis of FH in patients with a clinical presentation of IBS. The in vitro BAT based on CD63 detection on separate leukocytes 


Q3:
Please
supply
caption for
Figure 1

First part of the studv (a):
patients with a IBS diagnosis,
based on the Rome II criteria, who
undergone DBPC challenges for
suspected foods hypersensitivity

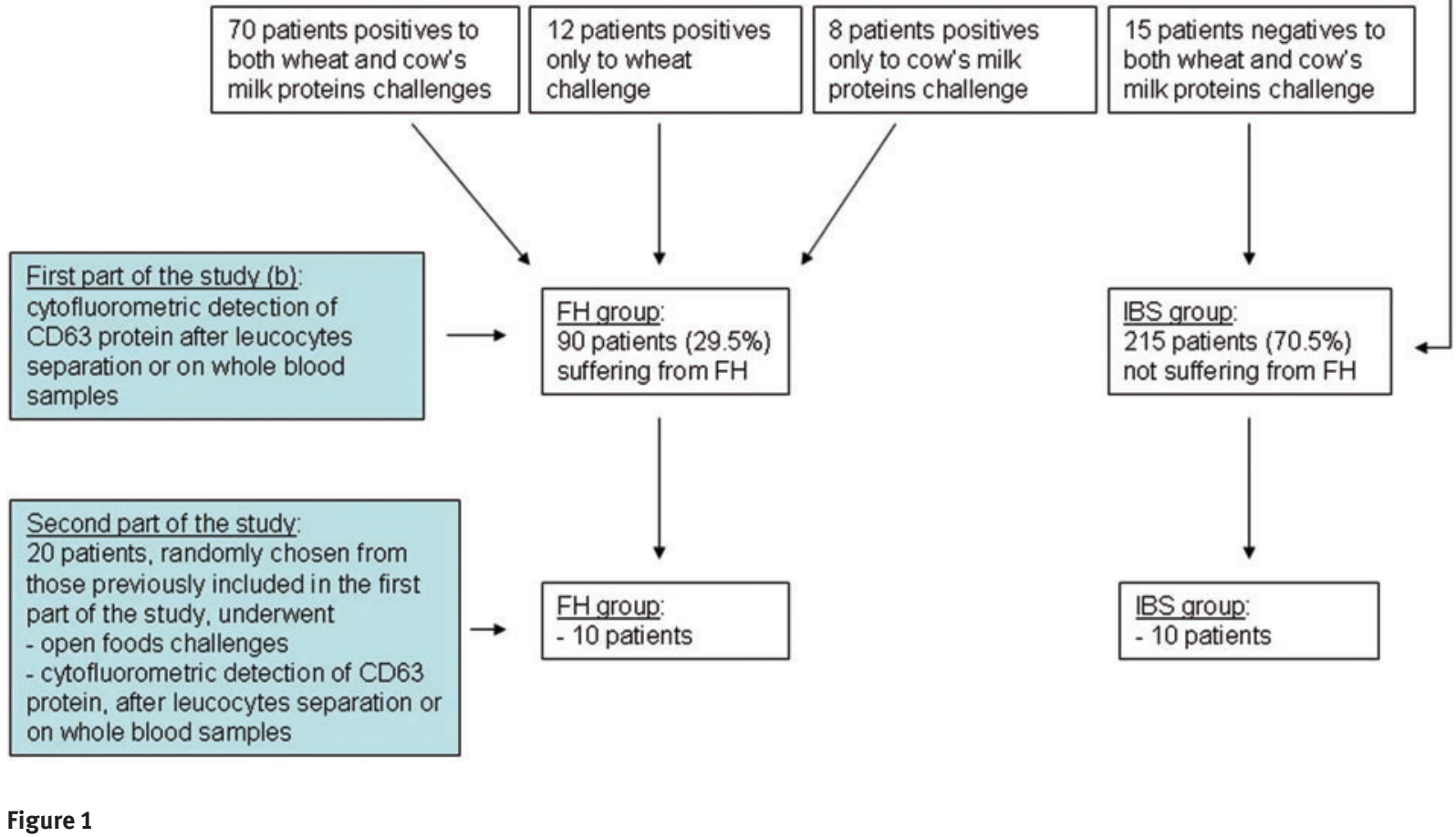

showed a higher sensitivity than the same assay on whole blood both in cow's milk protein- and wheat hypersensitivity diagnosis $(\mathrm{p}<0.0001)$. In fact, the sensitivity of the assay based on whole blood was very low. The specificity of the CD63 on separate leukocyte assay was also significantly higher than that of the assay based on whole blood ( $p=0.0004)$. As a consequence, the diagnostic accuracy in CMP hypersensitivity and in wheat hypersensitivity diagnoses was significantly higher for the CD63 assay

\begin{tabular}{llrr} 
Time period & BAT method & $\begin{array}{r}\text { Number } \\
\text { of IBS } \\
\text { diagnosis }\end{array}$ & $\begin{array}{r}\text { Number } \\
\text { of FH } \\
\text { diagnosis }\end{array}$ \\
\hline $\begin{array}{l}\text { January 2007- } \\
\text { March 2010 }\end{array}$ & CD 63 detection & 160 & 70 \\
$\begin{array}{l}\text { April 2010- } \\
\text { June 2011 }\end{array}$ & CD 203c detection & 55 & 20
\end{tabular}

Q4:

Table 1 is not cited in text. Please supply citation
Table 1 Number of patients studied according to the different periods and the BAT methods used.

Patients with a final FH diagnosis included cases with both wheat and cow's milk protein hypersensitivity and patients with hypersensitivity to one of these antigens. on separate leukocytes than for the assay on whole blood ( $p<0.0001$ for both CMP and wheat).

No statistical difference in sensitivity and specificity was observed for the diagnosis of CMP hypersensitivity considering the positive and negative results obtained with each different antigen used as in vitro stimulants - $\alpha$-lactalbumin, $\beta$-lactoglobulin, casein - both for the assay on separate leukocytes and on whole blood (data not shown).

\section{Second part of the study}

All 10 patients who were diagnosed as suffering from $\mathrm{FH}$ by DBPC challenge during the first part of the study and who were included in the prospective second part were still on the elimination diet at this time. In fact, they were well on the elimination diet and continued to suffer from IBS symptoms assuming wheat and/or foods containing cow's milk. During this part of the study, they underwent open food challenges which confirmed a persistent $\mathrm{FH}$ diagnosis. 


\section{Q5:}

+95 CI has

been rewritten as $+95 \%$ CI. Please check and amend if necessary

\begin{tabular}{lrr} 
& $\begin{array}{r}\text { CD63 on separate } \\
\text { leukocytes }\end{array}$ & $\begin{array}{r}\text { CD63 on } \\
\text { whole blood }\end{array}$ \\
\hline IBS unrelated to FH & $\mathrm{n}=160$ & $\mathrm{n}=55$ \\
Positive & 12 & 15 \\
Negative & 148 & 40 \\
IBS related to cow's milk & $\mathrm{n}=58$ & $\mathrm{n}=20$ \\
protein hypersensitivity & & \\
Positive & 50 & 3 \\
Negative & 8 & 17 \\
IBS related to wheat protein & $\mathrm{n}=62$ & $\mathrm{n}=20$ \\
hypersensitivity & & \\
Positive & 54 & 4 \\
Negative & 8 & 16 \\
\hline
\end{tabular}

Table 2 Results of the BAT based on CD63 detection on separate leukocytes and of the same assay on whole blood in the patients with IBS unrelated to FH and in those suffering with cow's milk protein (CMP) hypersensitivity and/or with wheat protein hypersensitivity.

aSeventy patients were positive to both wheat and cow's milk protein challenge, 12 patients were positive to wheat challenge only and eight patients were positive to cow's milk protein challenge only; bUpper normal limit for BAT was equal to the percentage of basophil activated after incubation with antigen less than three-fold the percentage of basophil activated spontaneously; 'For CMP hypersensitivity the following antigens were used in the BAT: $\alpha$-lactalbumin, $\beta$-lactoglobulin, casein; the positivity for one or more of the above antigens was considered indicative of "BAT positivity". For wheat hypersensitivity wheat proteins were used for the activation test.

$\begin{array}{rr}\text { CD63 assay } & \text { CD63 assay on } \\ \text { on separate } & \text { whole blood } \\ \text { leukocytes } & \end{array}$

All IBS patients $(\mathrm{n}=120)$

Sensitivity in cow's milk $\quad 86 \%(76 \%-93 \%) \quad 15 \%(6 \%-41 \%)$

hypersensitivity

Sensitivity in wheat protein $\quad 87 \%(77 \%-93 \%) \quad 25 \%(1 \%-50 \%)$

hypersensitivity

Specificity

Diagnostic accuracy in cow's $91 \%(86 \%-94 \%) \quad 57 \%(46 \%-68 \%)$

milk hypersensitivity

Diagnostic accuracy in

wheat hypersensitivity

$91 \%(86 \%-94 \%) \quad 59 \%(68 \%-86 \%)$

Table 3 Sensitivity, specificity and diagnostic accuracy $(+95 \% \mathrm{Cl})$ of the immunologic assays in the diagnosis of $\mathrm{FH}$ in patients with clinical presentation of IBS.

Sensitivity of CD63 assay on separate leukocytes versus CD63 on whole blood in CMP hypersensitivity: $p<0.0001$. Sensitivity of CD63 assay on separate leukocytes versus $C D 63$ on whole blood in wheat hypersensitivity: $p<0.0001$. Specificity of CD63 assay on separate leukocytes versus CD63 on whole blood: $p<0.0004$. Diagnostic accuracy of CD63 assay on separate leukocytes versus CD63 on whole blood in CMP hypersensitivity: $p<0.0001$. Diagnostic accuracy of CD63 assay on separate leukocytes versus CD63 on whole blood in wheat hypersensitivity: $p<0.0001$.
The other 10 patients with IBS, diagnosed as not suffering from FH during the first part of the study as they did not improve on the elimination diet or did not worsen on DBPC challenges, continued to consume every kind of food without restrictions.

Table 4 summarizes the results of the in vitro BAT performed with both methods. Using the old method based on CD63 detection after leukocyte separation we had found two false negative results in eight patients with wheat hypersensitivity and one false negative result in eight patients with CMP hypersensitivity. When we performed the new method based on CD63 detection on whole blood, we found a much lower concordance between DBPC challenge results and CD63 assay results. The specificity of both methods was high as no patients who were negative to the DBPC challenge were positive to both assays.

\section{Discussion}

Patients suffering from gastrointestinal symptoms and suspected FH very often have a difficult clinical history, characterized by many consultations and investigations without reaching any definite diagnosis. This is the clinical history, in particular, of the patients with IBS-like clinical manifestations [2]. In this respect, the possible relationship between IBS and gluten-sensitivity has been underlined very recently [13], although the pathogenesis of this condition still remains to be clarified and probably different subgroups of patients are wrongly included under an unique "gluten-sensitivity category" [10]. The major clinical problem in ascertaining whether patients with IBS really suffer from GS or FH is the lack of accurate diagnostic assays. These patients could have delayed reaction to foods, not IgE-mediated, and obviously none of the assays based on the IgE detection can be helpful in the diagnosis [3]. For this reason, in this kind of patient, the FH diagnosis must be based on the disappearance of symptoms on the elimination diet and a subsequent positive DBPC challenge. This diagnostic methodology has been used in our center for more than 15 years [14], and in the present study we ascertained that about one third of the IBS patients included were suffering from FH and were cured on the elimination diet. This percentage of FH in IBS was almost constant in the different cohorts we have studied in recent years [6, 7].

However, the problem of a simpler and less cumbersome diagnostic approach still exists. Very recently we have showed that in IBS-like patients, the assay based on the cytofluorometric detection of the in vitro basophil 


\begin{tabular}{|c|c|c|c|}
\hline Patients/sex/age & DBPC challenge results & CD63 assay on separate leukocytes & CD63 assay on whole blood \\
\hline n. $1 / F / 32$ years & Wheat positive/CMP positive & Wheat positive/CMP positive & Wheat negative/CMP negative \\
\hline n. $2 / F / 30$ years & Wheat positive/CMP positive & Wheat positive/CMP positive & Wheat negative/CMP negative \\
\hline n. $3 / F / 20$ years & Wheat positive/CMP positive & Wheat negative/CMP positive & Wheat negative/CMP positive \\
\hline n. $4 / F / 21$ years & Wheat positive/CMP negative & Wheat positive/CMP negative & Wheat negative/CMP negative \\
\hline n. $5 / F / 38$ years & Wheat positive/CMP positive & Wheat positive/CMP positive & Wheat positive/CMP negative \\
\hline n. $6 / F / 18$ years & Wheat positive/CMP negative & Wheat negative/CMP negative & Wheat negative/CMP negative \\
\hline n. $7 / F / 21$ years & Wheat negative/CMP positive & Wheat negative/CMP positive & Wheat negative/CMP positive \\
\hline n. $8 / F / 38$ years & Wheat negative/CMP positive & Wheat negative/CMP positive & Wheat negative/CMP negative \\
\hline n. $9 / F / 21$ years & Wheat positive/CMP positive & Wheat positive/CMP negative & Wheat negative/CMP negative \\
\hline n. $10 / F / 42$ years & Wheat positive/CMP positive & Wheat positive/CMP positive & Wheat positive/CMP negative \\
\hline n. $11 / F / 19$ years & Wheat negative/CMP negative & Wheat negative/CMP negative & Wheat negative/CMP negative \\
\hline n. $12 / \mathrm{M} / 38$ years & Wheat negative/CMP negative & Wheat negative/CMP negative & Wheat negative/CMP negative \\
\hline n. $13 / M / 45$ years & Wheat negative/CMP negative & Wheat negative/CMP negative & Wheat negative/CMP negative \\
\hline n. $14 / M / 21$ years & Wheat negative/CMP negative & Wheat negative/CMP negative & Wheat negative/CMP negative \\
\hline n. $15 / F / 49$ years & Wheat negative/CMP negative & Wheat negative/CMP negative & Wheat negative/CMP negative \\
\hline n. $16 / F / 38$ years & Wheat negative/CMP negative & Wheat negative/CMP negative & Wheat negative/CMP negative \\
\hline n. $17 / F / 18$ years & Wheat negative/CMP negative & Wheat negative/CMP negative & Wheat negative/CMP negative \\
\hline n. $18 / \mathrm{M} / 30$ years & Wheat negative/CMP negative & Wheat negative/CMP negative & Wheat negative/CMP negative \\
\hline n. $19 / \mathrm{M} / 20$ years & Wheat negative/CMP negative & Wheat negative/CMP negative & Wheat negative/CMP negative \\
\hline n. $20 / F / 21$ years & Wheat negative/CMP negative & Wheat negative/CMP negative & Wheat negative/CMP negative \\
\hline
\end{tabular}

Table 4 Comparison of BAT results (CD63 detection) performed after leukocyte separation and on whole blood in 10 patients positive to the DBPC challenges to wheat and/or CMP and in 10 patients negative to the DBPC challenges. False negative results (discordant towards the DBPC results) are marked in Bold.

activation (CD63 on separate leukocytes) by food antigens diagnosed FH with $86 \%$ sensitivity, $88 \%$ specificity, and $87 \%$ accuracy. This level of sensitivity was significantly higher than that of serum total IgE or food-specific IgE assays [7]. These data have determined an increased interest for the use of the cytofluorometric assay in FH and gluten-sensitive diagnoses and many colleagues searching for methodological details have written to us. However, the commercial assay which we had used in the past changed methods, and since 2010 a new assay on whole blood replaced the old assay performed on separated leukocytes. This inspired us to perform the present study to evaluate the diagnostic accuracy of the two different methods.

Our results clearly and surprisingly demonstrated that the new BAT based CD63 detection on whole blood samples did not work in FH diagnosis. The retrospective analysis performed on over 200 patients, all of whom underwent DBPC challenges, showed that whereas the old test confirmed very good sensitivity $(86 \%-87 \%)$ and specificity (91\%) in the FH diagnosis, the new test fell to a sensitivity of $15 \%-20 \%$. This astonishing lack of sensitivity and diagnostic accuracy in FH diagnosis was confirmed by the second part of the study, when we called back 20 patients who had been previously evaluated with the BAT method based on separated leukocytes and tested them with the new method performed on whole blood samples. In this case also, only two of eight patients with CMP hypersensitivity, or with wheat hypersensitivity, were positive to the new test.

It remains to be clarified what the reasons for such an evident fall in diagnostic accuracy could be. It has been pointed out that whole blood is less stable and functional than the isolated leukocytes, and this considerably reduces its range of use [15]. Furthermore, it has been demonstrated that the presence of allergen-specific IgG antibodies in the plasma may influence the results [15]. This could be an important factor in the patients we studied as the presence of anti-gliadin IgG antibodies in $50 \%-60 \%$ of gluten-sensitive, IBS-like patients has been demonstrated $[10,16]$ and we have demonstrated an evident overlap between patients "classified as gluten-sensitive" and those "classified as suffering from wheat hypersensitivity" [10]. Another factor which could negatively interfere with the accuracy of the new test is the reduced incubation time with the allergens, compared to the old test on separate leukocytes (15 min vs. $60 \mathrm{~min}$ ) [17]. Finally, a direct comparison between the technique on whole blood and that on isolated leukocytes has been reported and the latter appeared to be more sensitive for allergen-induced basophil CD63 activation [18]. 
It is evident that the main advantage of using whole blood in vitro basophil activation tests would be speed and simplicity of manipulation. However, our data strongly discouraged its use in FH diagnosis, and induced us to reconsider the use of the assay based on isolated leukocytes. The commercial assay currently available did not seem to be useful in the complicated diagnostic work-up of the suspected FH.

\section{References}

1. Boyce JA, Assa'ad A, Burks AW, Jones SM, Sampson HA, Wood RA, et al. Guidelines for the diagnosis and management of food allergy in the United States: Report of the NIAID-sponsored expert panel. J All Clin Immunol 2010;126:S1-58.

2. Bischoff S, Crowe SE. Gastrointestinal food allergy: new insights into pathophysiology and clinical perspectives. Gastroenterology 2005;128:1089-113.

3. Sampson HA, Sicherer SH, Birnbaum AH. AGA technical review on the evaluation of food allergy in gastrointestinal disorders. American Gastroenterological Association. Gastroenterology 2001;120:1026-40.

4. Niec AM, Frankum B, Talley NJ. Are adverse food reactions linked to irritable bowel syndrome? Am J Gastroenterol 1998;93:2184-90.

5. Atkinson W, Sheldon TA, Shaath N, Whorwell PJ. Food elimination based on IgG antibodies in irritable bowel syndrome: a randomised controlled trial. Gut 2004:53:1459-64.

6. Carroccio A, Brusca I, Mansueto P, Soresi M, D’Alcamo A, Ambrosiano G, et al. Fecal assays detect hypersensitivity to cow's milk protein and gluten in adults with irritable bowel syndrome. Clin Gastroenterol Hepatol 2011;9:965-70.

7. Carroccio A, Brusca I, Mansueto P, Pirrone G, Barrale M, Di Prima L, et al. A cytological assay for diagnosis of food hypersensitivity in patients with irritable bowel syndrome. Clin Gastroenterol Hepatol 2010;8:254-60.

8. Thompson WG, Longstreth GF, Drossman DA, Heaton KW, Irvine EJ, Müller-Lissner SA. Functional bowel disorders and functional abdominal pain. Gut 1999;45(Suppl 2):43-7.

9. Iacono G, Ravelli A, Di Prima L, Scalici C, Bolognini S, Chiappa $\mathrm{S}$, et al. Colonic lymphoid nodular hyperplasia in children: relationship to food hypersensitivity. Clin Gastroenterol Hepatol 2007;5:361-6.

\section{Conflict of interest statement}

Authors' conflict of interest disclosure: The authors stated that there are no conflicts of interest regarding the publication of this article. Research funding: None declared. Employment or leadership: None declared. Honorarium: None declared.

Received September 14, 2012; accepted October 16, 2012
10. Carroccio A, Mansueto P, Iacono G, Soresi M, D’Alcamo A, Cavataio $F$, et al. Non-celiac wheat sensitivity diagnosed by double-blind placebo-controlled challenge: exploring a new clinical entity. Am J Gastroenterol 2012 Jul 24. [Epub ahead of print].

11. Bruns DE, Huth EJ, Magid E, Young DS. Toward a checklist for the reporting of studies of diagnostic accuracy of medical tests. Clin Chem 2000;46:893-5.

12. Feinstein A. On the sensitivity, specificity and discrimination of diagnostic tests. Clin Pharmacol Ther 1975;17:104-10.

13. Verdu EF, Armstrong D, Murray JA. Between celiac disease and irritable bowel syndrome: the no man's land of gluten sensitivity. Am J Gastroenterol 2009;104:1587-94.

14. Iacono G, Cavataio F, Montalto G, Florena A, Soresi M, Tumminello M, et al. Intolerance of cow's milk and chronic constipation in children. N Engl J Med 1998;338:1100-4.

15. De Weck AL, Sanz ML, Gamboa PM, Aberer W, Bienvenu J, Blanca $M$, et al. Diagnostic tests based on human basophils: more potentials and perspectives than pitfalls. II. Technical issues. J Investig Allergol Clin Immunol 2008;18:143-55.

16. Volta U, Tovoli F, Cigola R, Parisi C, Fabbri A, Piscaglia M, et al. Serological tests in gluten-sensitivity. J Clin Gastroenterol 2012;46:680-5.

17. Hennersdorf F, Florian S, Jakob A, Baumgartner K, Sonneck K, Nordheim A, et al. Identification of CD13, CD107a, and CD164 as novel basophil-activation markers and dissection of two response patterns in time kinetics of IgE-depandent upregulation. Cell Res 2005;15:325-35.

18. De Weck AL, Gamboa P, Sanz ML. Comparison of two commercial basophil activation cytofluorometric tests for the diagnosis of allergies and pseudoallergies. EAACI Paris 2003;369-70 [abstract 1299]. 